\title{
Sylvain Laurens, Militer pour la science. Les mouvements rationalistes en France (1930-2005)
}

Jérôme Michalon

\section{OpenEdition}

Journals

Electronic version

URL: http://journals.openedition.org/lectures/35258

DOI: $10.4000 /$ lectures.35258

ISSN: 2116-5289

\section{Publisher}

Centre Max Weber

Brought to you by Centre national de la recherche scientifique (CNRS)

\section{Cnrs}

Electronic reference

Jérôme Michalon, «Sylvain Laurens, Militer pour la science. Les mouvements rationalistes en France (1930-2005) », Lectures [Online], Reviews, Online since 14 June 2019, connection on 12 November 2020. URL : http://journals.openedition.org/lectures/35258 ; DOI : https://doi.org/10.4000/lectures 35258

This text was automatically generated on 12 November 2020.

(c) Lectures - Toute reproduction interdite sans autorisation explicite de la rédaction / Any replication is submitted to the authorization of the editors 


\title{
Sylvain Laurens, Militer pour la science. Les mouvements rationalistes en France (1930-2005)
}

\author{
Jérôme Michalon
}

1 Alors que, dans plusieurs pays, sont organisées des « Marches pour les Sciences » visant à répondre tout autant au climato-scepticisme d'État, incarné par Donald Trump, qu'aux réductions drastiques des budgets alloués à la recherche et à l'enseignement supérieur, l'ouvrage de Sylvain Laurens parait à point nommé. Sociologue, maitre de conférences à l'EHESS, l'auteur se présente comme un «sociohistorien de l'État et des classes dominantes » (p. 9). Il n'avait jusqu'ici jamais travaillé sur les scientifiques, et encore moins sur l'engagement de ces derniers dans l'espace public. Ce sont ses précédentes enquêtes sur les pratiques de lobbying auprès de la Commission européenne qui l'ont aiguillé sur le rôle crucial joué par l'information scientifique dans les décisions politiques. C'est dans ce cadre que l'auteur a pris connaissance des organisations rationalistes, qui s'efforcent depuis plusieurs décennies de porter la parole des savants dans l'espace public, articulant ainsi un engagement « au nom de la science " et un engagement "pour la science». L'exploration de cette seconde dimension est au cœur de la recherche que Sylvain Laurens a entrepris en retraçant patiemment l'histoire des trois principales organisations rationalistes françaises (l'Union rationaliste l'Association française pour l'information scientifique et le mouvement zététique), depuis l'entre-deux guerres jusqu'au début des années 2000. Par le biais d'archives et de publications de ces associations, ainsi qu'au travers d'entretiens réalisés avec certains de leurs membres, l'auteur nous livre un récit très documenté de la genèse de ces collectifs, de leur évolution en lien avec l'histoire politique de la France $\mathrm{du} \mathrm{XX}^{\mathrm{e}}$ siècle, des propriétés sociales de leurs membres et de leur rapport avec d'autres organisations (syndicats, partis politiques, groupes de réflexion, groupes d'intérêt, industries, etc.). Il s'agit pour Sylvain Laurens de comprendre ce qui permet de constituer «la raison» ou "la défense de la science » comme argument politique, fédérant certains scientifiques, des intellectuels ou des "amateurs de science». L'auteur nous invite à suivre comment évolue la formulation de cet argument, en 
utilisant le terme d'«épistémologie» pour qualifier les valeurs défendues par les organisations rationalistes dans leur rapport au reste de la société.

2 À partir des six chapitres qui composent l'ouvrage, il est possible d'identifier trois grandes périodes, chacune correspondant à la prévalence d'une épistémologie particulière au sein de la mouvance rationaliste. Les deux premiers chapitres nous plongent ainsi dans ses racines, avec la création de l'Union rationaliste (UR) dans les années 1930, lieu de convergence de savants et d'intellectuels, pour beaucoup déjà liés par un engagement en faveur de Dreyfus, gravitant dans la galaxie de la «libre pensée ", de la franc-maçonnerie, attachés à une analyse critique de la religion, et affichant des sympathies pour le socialisme et le communisme. Composée très largement de physiciens et de biologistes (qui domineront pendant longtemps le mouvement rationaliste), l'UR est un espace où l'on débat des théories récentes d'Einstein et des derniers développements de la biologie darwinienne. Mais au-delà des discussions scientifiques, le discours soutient la nécessaire autonomie de l'activité scientifique vis-à-vis des pouvoirs religieux et étatiques (militaires notamment). Cette autonomie est défendue au nom de l'utilité sociale des sciences, perçues comme pouvant résoudre à peu près toutes les difficultés que l'époque rencontre. C'est à ce titre que Sylvain Laurens parle d'une "épistémologie engagée » pour qualifier le discours rationaliste d'alors: les sciences sont au service d'un projet humaniste de progrès social et d'émancipation collective qui mérite qu'on leur accorde « une place à part ». Le statut de chercheur est alors en création, avec le développement de la Caisse nationale des sciences, devenue le CNRS. L'UR et ses membres sont des acteurs de ce mouvement d'autonomisation de la recherche scientifique, entamé avant la Seconde Guerre mondiale et poursuivi à la Libération, donnant lieu à l'instauration durable d'une bureaucratie scientifique. L'UR se veut garante de cette autonomie nouvellement acquise, et à ce titre, revendique, dès sa création, une forme de " neutralité » politique. Cette neutralité affichée a permis à l'UR de survivre à la période de l'occupation allemande (tout en ayant fortement condamné le fascisme naissant), mais, aprèsguerre, elle a surtout pour intérêt de servir de "refuge" pour des scientifiques membres du PCF mais en désaccord avec le stalinisme et son instrumentalisation de la science (suite à l'affaire Lyssenko, notamment), mais aussi à des pacifistes convaincus, refusant de s'aligner sur l'un des deux camps émergeant avec la guerre froide. La neutralité de l'UR est aussi instrumentalisée par le PCF, qui a besoin de savants acquis à la cause mais pouvant s'exprimer à partir du lieu « neutre » que constitue l'association. Cette dernière permet également au parti de rentrer en contact avec les organisations «bourgeoises» (comme certaines loges maçonniques ou la Ligue des droits de l'homme), auxquelles les membres de l'UR participent par ailleurs.

3 Au tournant des années 1960, l'adhésion au "socialisme scientifique " s'érode fortement, à l'UR comme ailleurs, et les liens entre le PCF et l'association se distendent. À travers le lancement de la revue Raison présente, certains membres de l'UR cherchent encore à revivifier l'épistémologie engagée, en proposant des réflexions issues de la philosophie marxiste et des sciences humaines et sociales. Mais cette réorientation semble encore trop " politique » (ou plutôt encore trop proche du socialisme) au goût d'une partie des adhérents de l'UR. Pour ceux-ci, il est possible de séparer le projet rationaliste des valeurs humanistes qui l'avaient jusqu'ici porté, et de le redéfinir avant tout comme "la défense d'une méthode d'investigation spécifique pour saisir le réel » (p. 103). S'affirme alors une nouvelle épistémologie, l'« épistémologie expérimentale », 
portée par de nouveaux adhérents, souvent ingénieurs et médecins libéraux, soucieux de diffuser l'esprit scientifique auprès du " grand public ». L'apparition de cette figure un public non scientifique auquel s'adresser - vient alléger les tensions créées entre les deux camps de l'UR. Pour les uns, éduquer les masses pour les sortir de l'ignorance semble assez proche de l'idéal d'émancipation porté par le socialisme. Pour les autres, c'est un moyen de contrer l'exploitation marchande de l'attrait pour l'ésotérisme, le paranormal, l'ufologie et autres croyances populaires. Les années 1970 connaissent en effet un regain d'intérêt pour ces thématiques, dont témoigne le succès éditorial de nombreux ouvrages signés par des écrivains (parfois savants) utilisant des données et un style scientifiques. Soucieux de dénoncer l'utilisation de l'autorité scientifique à des fins irrationnelles et mercantiles, l'univers rationaliste va dès lors constituer la lutte contre les pseudosciences comme un nouveau cheval de bataille. C'est à travers la figure de l'Afis (Association française pour l'information scientifique) et de son fondateur, le journaliste Michel Rouzé, que Sylvain Laurens retrace cette nouvelle période du rationalisme français, qui se caractériser par la forte implication de " profanes », non scientifiques. "Citoyens engagés ", " amateurs de sciences ", issus de milieux sociaux variés, les adhérents de l'Afis et le lectorat de ses Cahiers, ont accès à des ressources scientifiques vulgarisées, simplifiées, pour pouvoir prendre part à la chasse aux astrologues, télépathes, mages, spirites ou magnétiseurs: autant de "charlatans » auxquels, entre autre chose, il est reproché de ne pas soumettre leurs " pouvoirs » à l'épreuve de l'expérimentation de laboratoire. Ce créneau sera radicalisé dans les années 1990 avec l'émergence de la Zététique, mouvement rationaliste dont l'une des particularités consiste à organiser des démonstrations publiques visant à reproduire les pratiques des "charlatans" pour en démontrer le caractère scientifiquement inepte ; l'épistémologie expérimentale atteint là son sommet.

Dans le sillage de cette lutte contre les pseudosciences, certains rationalistes s'attaquent à plusieurs courants d'idées à partir des années 1980. L'écologie figure en bonne place et fait l'objet de critiques virulentes, les associations proenvironnementales étant accusées d'alimenter des peurs irrationnelles contre l'innovation technologique, le progrès, voire contre le « réel » (pour reprendre le titre de l'ouvrage de Maurice Tubiana, Le Refus du réel, auquel Sylvain Laurens consacre plusieurs pages). La critique de la "technophobie" émerge alors dans les milieux rationalistes, portée par des personnes en interaction plus ou moins resserrée avec le monde industriel. Ceux-ci voient dans le développement technologique et industriel une nouvelle manière de diffuser le rationalisme et son esprit. Ainsi, au nom du rationalisme, sont publiés des textes qui défendent les pesticides, le développement du nucléaire et, plus tard, des OGM, les nanotechnologies ou le climato-scepticisme. Cette ligne est incarnée notamment par des individus qui naviguent professionnellement entre secteur privé et secteur public: ingénieurs formés aux "sciences appliquées ", travaillant dans des grandes entreprises publiques qui ont été privatisées (EDF-GDF, France Telecom), devenus pour certains des « managers "; savants formés à l'étranger, moins attachés à la recherche publique, habitués aux partenariats avec le monde industriel, et parfois devenus des "entrepreneurs ». Une "épistémologie de marché " monte en puissance dans la galaxie rationaliste, en particulier au sein de l'Afis qui, dans les années 2000, connaît un essor important tandis que l'UR décline considérablement. Alors qu'aujourd'hui se reconfigure (ou se délite, c'est selon) la bureaucratie scientifique, le mouvement rationaliste, qui a contribué à la créer, est plus que jamais divisé. Qu'il s'agisse des ennemis à combattre ou des valeurs au nom desquelles mener 
le combat, et alors même que la défiance institutionnelle vis-à-vis des sciences n'a sans doute jamais été aussi forte, les militants rationalistes ne parlent plus d'une seule voix, conclut Sylvain Laurens au terme de son analyse.

5 À travers cette véritable épopée du rationalisme français, Sylvain Laurens nous livre des éléments cruciaux sur la construction de l'autonomie scientifique. Au prisme de l'histoire des mouvements rationalistes, on comprend comment cette autonomie a pu se négocier entre d'une part des scientifiques unis derrière une définition de la science (au singulier) et de ses valeurs (à l'image de l'UR des débuts) et d'autre part des intérêts extra scientifiques (industriels, militaires ou politiques) puissants. Au fil des pages, on voit précisément cette autonomie se construire, être peu à peu remise en cause, à mesure que la bureaucratie scientifique s'installe, s'autonomisant elle-même des savants puis s'affaiblissant considérablement au profit d'un monde industriel qui internalise la recherche (tout en captant une partie des ressources publiques). Seule une perspective sociologique comme celle de l'auteur pouvait restituer ces fluctuations avec autant de finesse, et expliquer l'engagement rationaliste, au-delà des raisons avancées par les militants eux-mêmes. En cela, l'ouvrage de Sylvain Laurens montre que le geste initié par la sociologie des sciences, (en particulier celle des années 1970, dans le sillage des Science and Technology Studies) a encore de beaux jours devant lui. De la même manière que les STS ont montré l'intérêt d'une description de l'activité scientifique "de l'extérieur", la perspective sociologique adoptée ici se révèle extrêmement fructueuse en ce qu'elle parvient à donner du sens à cette association étrange entre un combat se présentant comme "dépolitisé » (en particulier par les tenants de l'épistémologie expérimentale) et une forme d'action empruntant beaucoup au registre militant. Comment devient-on militant pour la science? Le considérable travail de Sylvain Laurens y répond sans aucun doute et ouvre des perspectives inédites pour une sociologie politique des sciences, qui s'est pour l'instant plutôt concentrée sur l'enrôlement des scientifiques derrière certaines causes, ou sur la dimension engagée du travail de recherche en lui-même.

\section{AUTHOR}

\section{JÉRÔME MICHALON}

Chargé de recherche au CNRS, au laboratoire Triangle (UMR 5206), Jérôme Michalon est sociologue, et travaille sur les rapports humains/animaux, dans une perspective de sociologie des sciences et de sociologie des mobilisations. 